\title{
Closing Emergency Operating Rooms Improves Efficiency
}

\author{
Gerhard Wullink • Mark Van Houdenhoven • \\ Erwin W. Hans • Jeroen M. van Oostrum • \\ Marieke van der Lans • Geert Kazemier
}

Received: 31 May 2007 / Accepted: 21 August 2007 / Published online: 19 September 2007

(C) Springer Science + Business Media, LLC 2007

\begin{abstract}
Long waiting times for emergency operations increase a patient's risk of postoperative complications and morbidity. Reserving Operating Room (OR) capacity is a common technique to maximize the responsiveness of an OR in case of arrival of an emergency patient. This study determines the best way to reserve OR time for emergency surgery. In this study two approaches of reserving capacity were compared: (1) concentrating all reserved OR capacity in dedicated emergency ORs, and (2) evenly reserving capacity in all elective ORs. By using a discrete event simulation model the real situation was modelled. Main outcome measures were: (1) waiting time, (2) staff overtime, and (3) OR utilisation were evaluated for the two approaches. Results indicated that the policy of reserving capacity for emergency surgery in all elective ORs led to an improvement in waiting times for emergency
\end{abstract}

G. Wullink · M. Van Houdenhoven ·

J. M. van Oostrum $(\bowtie) \cdot$ G. Kazemier

Department of Operating Rooms,

Erasmus University Medical Centre,

P.O. Box 2040, 3000CA Rotterdam, the Netherlands

e-mail: j.vanoostrum@erasmusmc.nl

G. Wullink $\cdot$ M. Van Houdenhoven •

J. M. van Oostrum

Department of Intensive Care,

Erasmus University Medical Center,

Rotterdam, the Netherlands

G. Kazemier

Department of Surgery,

Erasmus University Medical Center,

Rotterdam, the Netherlands

E. W. Hans $\cdot$ M. van der Lans

School of Business, Public Administration and Technology,

University of Twente,

P.O. Box 217, 7500AE, Enschede, the Netherlands surgery from $74( \pm 4.4)$ minutes to $8( \pm 0.5)$ min. Working in overtime was reduced by $20 \%$, and overall OR utilisation can increase by around 3\%. Emergency patients are operated upon more efficiently on elective Operating Rooms instead of a dedicated Emergency OR. The results of this study led to closing of the Emergency OR in the Erasmus MC (Rotterdam, The Netherlands).

Keywords Operating room · Emergency surgery · Health care efficiency · Discrete-event simulation

\section{Introduction}

Postponing emergency surgery may increase a patient's risk of postoperative complications and morbidity. Waiting times depend on the speed at which an operating room (OR) can organize its resources to operate upon an emergency patient. A common approach to deal with emergency procedures is to reserve OR capacity; this is believed to increase responsiveness to the arrival of an emergency patient $[1,2]$.

There are two basic policies for reserving OR capacity for emergency patients: in dedicated emergency ORs or in all elective ORs. The first policy, reserving capacity in dedicated emergency ORs, would combine short waiting times with low utilisation of expensive OR capacity. Hence, it is an expensive option, since one or more entire ORs cannot be used for elective surgery. Emergency patients arriving at a hospital that has adopted the first policy will be operated immediately if the dedicated OR is empty and will have to queue otherwise, whereas patients arriving at a hospital that has adopted the second policy can be operated once one of the ongoing elective cases has ended. Other planned cases will then be postponed to allow the 
Table 1 Aggregate descriptive statistics of the OR in Erasmus MC

\begin{tabular}{ll}
\hline Description & Number \\
\hline Number of different surgical procedure types & 328 \\
Mean number of elective cases per day & 32 \\
Mean case duration (minutes) & 142 \\
Standard deviation of the case duration (minutes) & 45 \\
Mean number of emergency cases per day & 5 \\
Mean emergency case duration (minutes) & 126 \\
Standard deviation of emergency case duration (minutes) & 91 \\
\hline
\end{tabular}

emergency operation. Thus, besides influencing waiting times of emergency patients, the choice of either policy will have impact on the amount of overtime and OR utilisation.

Little evidence is available on the performance in terms of waiting times, OR utilisation, and overtime for the policy of reserving capacity for emergency patients in all elective ORs. In this study we determined the best policy to reserve time for emergency patients. We assessed the policies using a discrete-event simulation model for this purpose.

\section{Data and methods}

Erasmus MC with 1,300 beds is the largest teaching hospital and tertiary referral centre in the Netherlands. It provides for the complete spectrum of surgical procedures, including transplantation and trauma surgery. Of the 34,500 admissions per year, some 20,000 involve a surgical procedure. Data on more than 180,000 surgical procedures have prospectively been collected since 1994, including procedure duration, the procedure name, the procedure type (elective or emergency), and surgical specialty involved. Data had been approved immediately after the surgical procedure by the surgery or anaesthesia nurse. The duration of surgical procedures, both emergency and elective, is assumed to be lognormal [3]. Table 1 shows the aggregate descriptive statistics of the central OR department of the Erasmus MC.

A block planning approach to schedule the elective procedures was assumed [4, 5]. We assumed that on average 12 ORs per day, five days per week were staffed and available. The availability of the staffed ORs was limited to $450 \mathrm{~min}$ per day. Moreover, all ORs were assumed to be multi-functional, i.e., all procedures types can be performed in all ORs.

We developed a discrete event simulation model [6, 7], using the simulation software tool eM-Plant (Plano, USA). This simulation model was a representation of the Erasmus MC 12 OR set-up. We simulated days independently of each other. In the first emergency policy, with emergency capacity allocated to one dedicated emergency $\mathrm{OR}$, the remaining free OR time is allocated to exclusively elective ORs. In the second policy, with emergency time allocated to each elective OR, the reserved OR time is distributed evenly over all elective ORs. Figure 1 illustrates these policies.

A schedule with elective surgical cases is the input for the simulation model. These schedules are constructed by applying a first-fit algorithm [8]. The first-fit algorithm subsequently assigns for each surgical department separately surgical cases to the first available OR. The resulting surgical case schedule specifies therefore for each OR the elective surgical procedures to be performed. Procedures are planned using their mean duration, based upon the available data.

The given elective OR program forms the starting point for the comparison. We model the duration of elective producers by a procedure-specific lognormal distribution. Emergency patients arrive according to a Poisson process (with mean inter-arrival time of $1 / 5$ day): inter-arrival times are mutually independent and exponentially distributed. The duration of emergency surgery was based upon one lognormal distribution for all emergency procedures together. Emergency operation is on a first-come-first-served basis and is performed either after the first completion of an elective operation or at the emergency OR, depending on the policy adopted. Each specialty in Erasmus MC reserves one surgeon for emergency surgery. In practice, that particular day this surgeon has no outpatient clinics, teaching activities, or scheduled elective surgery, but typically administrative and research activities. We mod-
Fig. 1 Visualization of the two studied policies for allocating reserved OR time

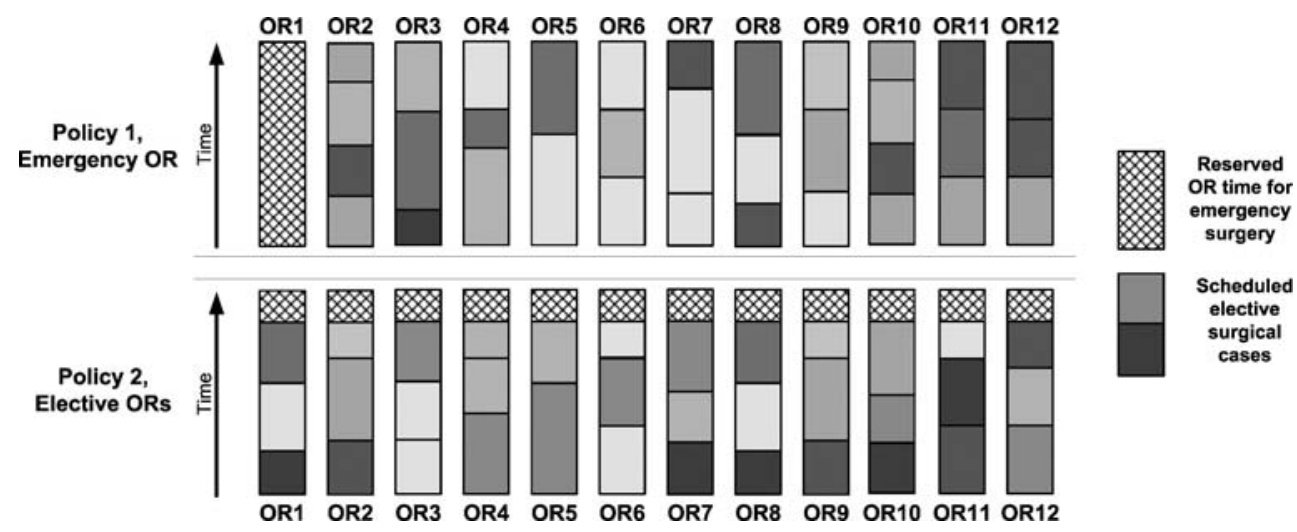


elled no delay in starting emergency surgery due to surgeon or OR staff unavailability. We modelled no delay in starting emergency surgery due to surgeon or OR staff unavailability. Elective procedures planned in an OR are postponed until after the emergency operation and might be executed in overtime.

Overtime is defined as the time used for surgical procedures after the regular block time has ended. Efficiency of OR utilisation is calculated as the ratio between the total used operating time for elective procedures and the available time. The sequential procedure [9] to determine the run length of the simulation with a maximum deviation $10 \%$ and a reliability of $90 \%$ yielded a run length of 780 days, which includes approximately 4,000 emergency patients.

\section{Results}

Waiting times are plotted cumulatively in Fig. 2. In policy 1 , with use of a dedicated emergency OR, all 4,000 emergency patients were operated on within $7 \mathrm{~h}$. The mean waiting time was $74( \pm 4.4) \mathrm{min}$. In policy 2 , with capacity for emergency surgery allocated to all elective ORs, all 4,000 emergency patients were operated upon within $80 \mathrm{~min}$. The mean waiting time was $8( \pm 0.5) \mathrm{min}$.

Table 2 shows values for the other two performance indicators broken down for type of policy. Efficiency of OR utilisation computed for all ORs in the first policy is $74 \%$; for the second policy it is $77 \%$. Overall, the second policy, with emergency capacity allocated to all elective ORs, substantially outperforms the first policy, with a dedicated emergency OR, on all outcome measures.

\section{Discussion}

This study showed that reserving capacity for emergency surgery in elective ORs performs better than the policy of a dedicated OR for emergency procedures in a large teaching

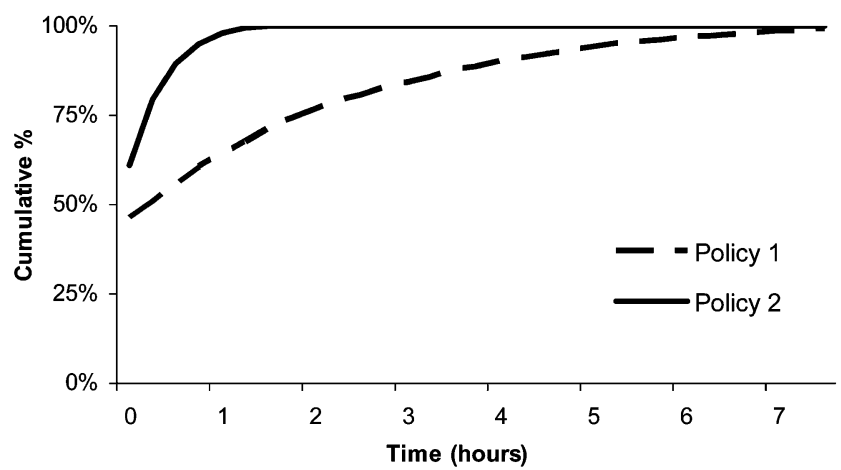

Fig. 2 Cumulative percentage of emergency patients in the two studied policies, treated within a certain (waiting) interval
Table 2 Overview results of the outcome measures

\begin{tabular}{lll}
\hline Emergency policy: & Policy 1 & Policy 2 \\
\hline Total overtime per day (hours) & 10.6 & 8.4 \\
$\begin{array}{l}\text { Mean number of ORs with overtime } \\
\text { per day }\end{array}$ & 3.6 & 3.8 \\
$\begin{array}{l}\text { Mean emergency patients' waiting } \\
\text { time (minutes) }\end{array}$ & $74( \pm 4.4)$ & $8( \pm 0.5)$ \\
OR utilization $^{\mathrm{a}}(\%)$ & 74 & 77 \\
\hline
\end{tabular}

${ }^{\text {a }}$ The OR utilisation is the ratio of elective surgery hours performed and the available capacity

hospital, based on a discrete-event simulation study with the three performance indicators: waiting time, overtime, and cost effectiveness of the OR.

The policy of allocating OR capacity for emergency surgery to elective ORs requires the OR department to be flexible. Upon arrival of an emergency patient, one of the ORs will have to fit the emergency operation into the elective OR schedule. The patients originally planned will have to be operated on either in another OR or at a later time. This requires flexibility of OR staff and surgeons in dealing with and accepting frequent changes to the original elective surgical case schedule. Also it requires OR to be equipped for all kinds of emergency surgery. Although OR departments that have physical overcapacity, i.e. OR departments where in general some of the ORs are unused, do not face this problem as they may allocate the emergency patient to an empty room that is sufficiently equipped. This way the OR staff have to move to this room, but not all rooms need to be fully equipped for all emergency surgery.

Interrupting the execution of the elective surgical case schedule for emergency patients may substantially delay elective cases. However, inpatients are typically admitted to a ward before they are brought to the OR. Although delay due to emergency arrivals may cause inconvenience of patients it does not disturb processes in the OR.

Besides reserving OR capacity for emergency patients, ORs generally need to reserve capacity to cope with the variability in the session durations. In the elective policy, reservation might be shared to increase the flexibility for dealing with unexpected long case duration and emergency surgery, whereas the dedicated policy does not offer the opportunity to use this overflow principle.

In OR departments that have dedicated emergency ORs it is common practice to re-assign staff to elective ORs to deal with temporary staff shortages. Hence, upon arrival of an emergency patient, the team may be incomplete, which implies the patient must wait until the team is complete again, typically when one of the ongoing elective cases ends. This practice considerably reduces the advantage of a dedicated OR. 
A dedicated emergency OR may cause queuing of emergency patients, confronting OR management and surgeons with the question which patient should be operated on first. Since such decisions are typically based on medical urgency, trauma procedures or a ruptured abdominal aneurysm will often be given preference over, for instance, fracture surgery. Hence, surgeries of specialties with less acute cases are more likely to be postponed. This would be less so if capacity for emergency surgery were to be allocated to all elective ORs, providing for various emergency patients to be operated on simultaneously.

Implementation of the policy by which emergency capacity is reserved in all elective ORs, requires all stakeholders on the OR to strictly adhere to the policy. In fact, the surgical departments that use a single OR face the so-called prisoner's dilemma. A single surgical department may benefit from not reserving capacity for emergency surgery, whereas this is disadvantageous for all surgical departments together. If one or more surgical departments do not reserve free OR capacity on their own ORs and hence must use reserved capacity of other specialties, the latter face the prisoner's dilemma. Successful implementation, therefore, would require dedication of all surgical departments.

In this study we have chosen to adopt discrete-event simulation to assess both policies, while application of queuing theory might have been another method to compare both policies. Application of queuing theory to the problem at hand is, however, not straightforward due to the probability distribution of surgery duration. Further, ongoing research might show application of queuing theory to the problem addressed in this paper.

In conclusion, we have compared two policies to reserve OR capacity for emergency surgery. Results obtained from a discrete-event simulation study show that distribution of free OR capacity evenly over all elective ORs performs better than dedicated ORs on measures reflecting quality of patient care, staff satisfaction, and cost-effectiveness. The policy of reserving free capacity can be successfully implemented on ORs only if all stakeholders were to participate. Moreover, besides the quantitative benefits as shown in this paper, it offers several, more soft advantages to improve ways of dealing with the variability that is inherent to medical processes.

\section{References}

1. Gallivan, S., Utley, M., Treasure, T., and Valencia, O., Booked inpatient admissions and hospital capacity: mathematical modelling study. BMJ 324:7332280-282, 2002.

2. Hopp, W. J., and Spearman, M. L., Factory physics-foundations of manufacturing management. Boston: IRWIN, 1996.

3. Strum, D. P., May, J. H., and Vargas, L. G., Modeling the uncertainty of surgical procedure times: comparison of log-normal and normal models. Anesthesiology 92:41160-1167, 2000.

4. Strum, D. P., Vargas, L. G., and May, J. H., Surgical subspecialty block utilization and capacity planning: a minimal cost analysis model. Anesthesiology 90:41176-1185, 1999.

5. Ozkarahan, I., Allocation of surgical procedures to operating rooms. J. Med. Syst. 19:4333-352, 1995.

6. Jun, J. B., Jacobson, S. H., and Swisher, J. R., Application of discrete-event simulation in health care clinics: A survey. J. Oper. Res. Soc. 50:2109-123, 1999.

7. Costa, A. X., Ridley, S. A., and Shahani, A. K., Mathematical modelling and simulation for planning critical care capacity. Anaesthesia 58:320-327, 2003.

8. Hans, E. W., Wullink, G., Houdenhoven, M., and Kazemier, G. Robust surgery loading. Eur. J. Oper. Res., 2007. DOI 10.1016/ j.ejor.2006.08.022

9. Law, A. M., and Kelton, W. D., Simulation modeling and analysis. Singapore: McGraw-Hill, 2000. 